\title{
Forces inside the nucleon on the light front from 3D Breit frame force distributions: Abel tomography case
}

\author{
Julia Yu. Panteleeva ${ }^{1}$ and Maxim V. Polyakov $\oplus^{1,2}$ \\ ${ }^{1}$ Ruhr University Bochum, Faculty of Physics and Astronomy, Institute for Theoretical Physics II, \\ D-44870 Bochum, Germany \\ ${ }^{2}$ Petersburg Nuclear Physics Institute, Gatchina, 188300 St. Petersburg, Russia
}

(Received 4 March 2021; accepted 15 June 2021; published 9 July 2021)

\begin{abstract}
We derive simple relations which express 2D light front force distributions in terms of 3D Breit frame pressure and shear force distributions. Mathematically the relations correspond to invertible Abel transformation and they establish one-to-one mathematical equivalence of 3D Breit frame force distributions and 2D light front ones. The beauty and simplicity of the Abel transformations allow us to derive a number of new relations for light front force distributions. Any knowledge (model calculation, experimental measurement, etc.) about pressure and shear force distributions in Breit frame can be unambiguously transformed into light front force distributions with the help of Abel transformation. Due to the positivity property of the Abel transformation we obtain an important result - the 2D local stability conditions are satisfied automatically if the corresponding 3D stability conditions are fulfilled. In this way we established not only mathematical equivalence of $2 \mathrm{D}$ and $3 \mathrm{D}$ forces distributions but also their physics equivalence in terms of stability. As an illustration of how the relations work, we calculated the light front force distributions for a large nucleus as a liquid drop, and for large $N_{c}$ nucleon as a chiral soliton.
\end{abstract}

DOI: 10.1103/PhysRevD.104.014008

\section{INTRODUCTION}

The linear response of a hadron to a change of the external space-time metric is described by the gravitational form factors (GFFs). The GFFs contain rich information about the internal structure of hadrons, for a detailed review see, e.g., Ref. [1]. Particular interest for us here are the mechanical properties-elastic pressure and shear force distributions inside the hadron of a mass $m$. These distributions can be characterized by the Breit frame (BF) static energy momentum tensor (EMT) [2] $]^{1}$ :

$$
\Theta^{\mu \nu}(\vec{r})=\int \frac{d^{3} \Delta}{(2 \pi)^{3} 2 E} e^{-i \vec{\Delta} \cdot \vec{r}}\left\langle p^{\prime}\left|\hat{\Theta}_{\mathrm{QCD}}^{\mu \nu}(0)\right| p\right\rangle
$$

\footnotetext{
${ }^{1}$ Although it is not necessary for derivations here, we note that for the distances $r \gg 1 /(2 m)$ the Breit frame static EMT has clear probabilistic interpretation, for the distances $\sim 1 /(2 m)$ it can be viewed as quasiprobabilistic phase-space average (in Wigner sense) of the observables in the rest frame of the hadron. Detailed discussion of this issue can be found in Ref. [3]. We stress that the interpretation of the BF static EMT is not relevant for the derivation here.

Published by the American Physical Society under the terms of the Creative Commons Attribution 4.0 International license. Further distribution of this work must maintain attribution to the author(s) and the published article's title, journal citation, and DOI. Funded by SCOAP ${ }^{3}$.
}

Here $\hat{\Theta}_{\mathrm{QCD}}^{\mu \nu}(0)$ is the QCD energy-momentum tensor (EMT) operator the matrix element of which is computed between hadron states with momenta $p^{0}=p^{0 \prime}=E=$ $\sqrt{m^{2}+\vec{\Delta}^{2} / 4}$, and $p^{i \prime}=-p^{i}=\Delta^{i} / 2$. The 00 component of the static EMT contains the information about the energy distribution inside the hadron, $0 i$ component about the spin distribution, and $i k$ components provide us the distribution of elastic pressure and shear forces inside the hadron [2].

In relativistic quantum field theory it is impossible to localize one-particle state with accuracy better than its Compton wave length $\lambda=\frac{\hbar}{m c}$. Therefore, at the distances smaller or of the order of $\chi$ one has to interpret the Breit frame static EMT (1) from a quasiprobabilistic phase-space perspective $[3,4]$. For the first time the phase-space picture to connect BF to light front was in the study of the angular momentum distribution [5]. The static EMT (1) can be viewed as the Wigner phase-space average $[6,7]$ of the force distributions inside the rest frame nucleon. See detailed discussion in Ref. [4] for EMT and Ref. [3] for the charge densities. Due to Heisenberg's uncertainty principle the Wigner distributions have only quasiprobabilistic interpretation. In the nonrelativistic limit [which corresponds to distances $r \gg \frac{1}{2 m}$ in Eq. (1)] the Wigner distributions acquire strict probabilistic interpretation, see detailed discussion in [3-5]. 
If one insists on strict probabilistic interpretation of distributions, the static EMT (1) for $r \sim 1 /(2 m)$ acquires the so-called relativistic corrections discussed since 1950th [8], for more recent discussion see Refs. [9-11]. The relativistic corrections can be kinematically suppressed if one considers the distributions in the infinite momentum frame (IMF) or if one uses quantization on the light front, see, e.g., Refs. [9,10]. In a recent paper [3], using the phase-space Wigner distributions, the natural interpolation between the Breit frame and IMF charge distributions was obtained, such analysis can be repeated for the case of force distributions.

The densities for the internal force distributions in the nucleon in IMF and on light front were derived first in Ref. [4] with help of Wigner phase-space distribution. More recently, light front force distributions were also obtained in Ref. [12] using methods of the light cone quantization. These densities have strict probabilistic interpretation (no relativistic corrections) and they are identical in both approaches. The corresponding densities are twodimensional (2D) and are distorted relative 3D quasiprobabilistic distributions due to the motion of the nucleon relative to observer.

Our aim here is to relate the 2D light front densities of internal forces inside the hadron to the 3D Breit frame distributions in a unequivocal way. We derive one-to-one relations between light front and Breit frame distributions: any result (model calculation, experimental measurement, etc.) for 3D Breit frame forces can be unambiguously translated to the form of the 2D light front force distributions (and vice versa). The corresponding relations are given by invertible Abel transformation which preserve the positivity properties of the force distributions. The latter property is very important as it allows us to make conclusions about the stability of the system working with 3D Breit frame distributions without worrying about possible relativistic corrections.

Usually it is very difficult to compute directly the 2D light front force distributions in various dynamical models of the hadron structure. Moreover, some of the models (e.g., bag models, constituent quark models, liquid drop models of heavy nuclei, etc.) can be formulated only in the rest frame of the hadron, and the direct calculation of the 2D densities is impossible. The obtained here unequivocal $2 \mathrm{D} \leftrightarrow 3 \mathrm{D}$ correspondence allows one to obtain $2 \mathrm{D}$ light front force distributions in such class of the models, and it simplifies considerably the corresponding calculations in relativistically covariant models (e.g., in the Skyrme model). This extends considerably the "toolkit" for studies of light front force distributions. This feature of our approach is illustrated in Secs. III and IV in which, using the Abel transformation, we compute 2D light front force distributions inside a large nucleus and in the nucleon using the liquid drop model and the Skyrme model correspondingly, thus providing the first dynamical calculation of the light front force distributions in hadrons.
Another aspect, where the advantage of the Abel transformation is clearly seen, is that the 3D Breit frame force distributions are more intuitive- there are many theoretical methods (see, e.g., methods for analysis of stability of the astrophysical objects in $[13,14])$ to analyze mechanical properties of various physics system using the 3D force distributions in the rest frame of the considered system. The relations between 3D and 2D force distributions derived here allow us to applied these well developed methods to analysis of the light front force distributions. The key for that possibility is the positivity property of the Abel transformation.

\section{RELATIONS BETWEEN 3D BREIT FRAME AND 2D LIGHT FRONT FORCE DISTRIBUTIONS}

For the nucleon there are three independent EMT form factors $[15,16]^{2}$ :

$$
\begin{aligned}
\left\langle p^{\prime}\left|\hat{\Theta}_{\mathrm{QCD}}^{\mu \nu}(0)\right| p\right\rangle= & \bar{u}\left(p^{\prime}\right)\left[A(t) \frac{P^{\mu} P^{\nu}}{m}+J(t) \frac{i P^{\{\mu} \sigma^{\nu\} \alpha} \Delta_{\alpha}}{m}\right. \\
& \left.+\frac{D(t)}{4 m}\left(\Delta^{\mu} \Delta^{\nu}-\eta^{\mu \nu} \Delta^{2}\right)\right] u(p),
\end{aligned}
$$

where $\hat{\Theta}_{\mathrm{QCD}}^{\mu \nu}(x)$ is the symmetric EMT operator of QCD, $P=\left(p+p^{\prime}\right) / 2, \Delta=p^{\prime}-p, t=\Delta^{2}$, and symmetrization operation is defined as $X_{\{\mu} Y_{\nu\}}=\frac{1}{2}\left(X_{\mu} Y_{\nu}+X_{\nu} Y_{\mu}\right)$. The values of the nucleon EMT form factors at zero momentum transfer provide us with three basic mechanical characteristics of the nucleon-the mass $m$, the spin $J=1 / 2$, and the $\mathrm{D}$ (ruck)-term $D(0)$. While the mass and spin of the nucleon are well-studied and well-measured quantities, the third mechanical characteristics-the Druck term or $D$-term-is more subtle, as it is related to the distribution of the internal forces inside the nucleon [2]. Important distinguishing feature of the nucleon $D$-term is that to access it one needs variations of the space-time metric such that the resulting Riemann tensor is nonzero. This feature of the Druck term is especially clearly seen in terms of effective field theory for pions and nucleon in curved space discussed recently in details in Ref. [18]. To access the mass and the spin it is enough to perform the variation of the metric with zero Riemann tensor, e.g., to go to a noninertial coordinate system. Despite the difficulty to access the Druck form factor, the first experimental data are available for the nucleon [19-21] and for the pion [22]. However the systematic and statistical uncertainties are still large. This will be considerably improved with new experiments on hard exclusive processes.

The Breit frame distributions of the elastic pressure $p(r)$ and shear force $s(r)$ in 3D can be obtained in terms of Druck form factor $D(t)$ through [1,2]:

\footnotetext{
${ }^{2}$ For definition of the gravitational form factors for hadrons of arbitrary spin see Ref. [17].
} 


$$
\begin{aligned}
s(r) & =-\frac{1}{4 m} r \frac{d}{d r} \frac{1}{r} \frac{d}{d r} \tilde{D}(r), \quad p(r)=\frac{1}{6 m} \frac{1}{r^{2}} \frac{d}{d r} r^{2} \frac{d}{d r} \tilde{D}(r) \\
\tilde{D}(r) & =\int \frac{d^{3} \boldsymbol{\Delta}}{(2 \pi)^{3}} e^{-i \Delta \mathbf{r}} D\left(-\Delta^{2}\right)
\end{aligned}
$$

Recently in Refs. $[4,12]$ the 2D light front pressure and shear force distributions were obtained in terms of the same Druck form factor $D(t)$ (we follow closely the notations of Ref. [12].):

$$
\begin{aligned}
\tilde{D}\left(x_{\perp}\right) & =\frac{1}{4 P^{+}} \int \frac{d^{2} \boldsymbol{\Delta}_{\perp}}{(2 \pi)^{2}} D\left(-\Delta_{\perp}^{2}\right) e^{-i \mathbf{\Delta}_{\perp} \cdot \mathbf{x}_{\perp},} \\
p^{(2 \mathrm{D})}\left(x_{\perp}\right) & =\frac{1}{2 x_{\perp}} \frac{d}{d x_{\perp}}\left(x_{\perp} \frac{d}{d x_{\perp}} \tilde{D}\left(x_{\perp}\right)\right), \\
s^{(2 \mathrm{D})}\left(x_{\perp}\right) & =-x_{\perp} \frac{d}{d x_{\perp}}\left(\frac{1}{x_{\perp}} \frac{d}{d x_{\perp}} \tilde{D}\left(x_{\perp}\right)\right),
\end{aligned}
$$

where $\mathbf{x}_{\perp}$ is the $2 \mathrm{D}$ vector in the transverse plane. Identical up to a (conventional) global rescaling by $P^{+} / m$ equations were derived in Ref. [4], using Wigner phase-space distribution in IMF.

As the 2D and 3D force distributions are expressed in terms of the same Druck form factor $D(t)$, they can be easily related to each other. Below we give these relations. For convenience we introduce $2 \mathrm{D}$ pressure $\mathcal{P}\left(x_{\perp}\right)$ and shear force distribution $\mathcal{S}\left(x_{\perp}\right)$ which differ from those in Eq. (5) by multiplication with the Lorentz factor $\frac{P^{+}}{2 m}$ :

$\mathcal{S}\left(x_{\perp}\right)=\frac{P^{+}}{2 m} s^{(2 \mathrm{D})}\left(x_{\perp}\right), \quad \mathcal{P}\left(x_{\perp}\right)=\frac{P^{+}}{2 m} p^{(2 \mathrm{D})}\left(x_{\perp}\right)$.

These 2D force distributions can be easily obtained in terms of 3D distributions defined in Eq. (3):

$$
\begin{aligned}
\mathcal{S}\left(x_{\perp}\right) & =\int_{x_{\perp}}^{\infty} \frac{d r}{r} s(r) \frac{x_{\perp}^{2}}{\sqrt{r^{2}-x_{\perp}^{2}}} \\
\frac{1}{2} \mathcal{S}\left(x_{\perp}\right)+\mathcal{P}\left(x_{\perp}\right) & =\int_{x_{\perp}}^{\infty} \frac{d r}{r} s(r) \sqrt{r^{2}-x_{\perp}^{2}} .
\end{aligned}
$$

These equations have the form of invertible Abel transformation [23], this is a key observation in the present paper. The functions $\mathcal{S}\left(x_{\perp}\right) / x_{\perp}^{2}$ is the Abel image of $s(r)$. For the readers' convenience we collected basic facts about the Abel transformation in the Appendix.

The Eqs. (7) can be obtained from analogous equations in Ref. [4] by change of the integration variable. The inverse transformation was not discussed in [4], we provide it in the present paper. Using the fact that Eqs. (7) correspond to Abel transformation it can be easily obtained their inversion:

$$
\begin{aligned}
s(r) & =-\frac{2}{\pi} r^{2} \int_{r}^{\infty} d x_{\perp} \frac{d}{d x_{\perp}}\left(\frac{\mathcal{S}\left(x_{\perp}\right)}{x_{\perp}^{2}}\right) \frac{1}{\sqrt{x_{\perp}^{2}-r^{2}}} \\
\frac{2}{3} s(r)+p(r) & =\frac{4}{\pi} \int_{r}^{\infty} \frac{d x_{\perp}}{x_{\perp}} \mathcal{S}\left(x_{\perp}\right) \frac{1}{\sqrt{x_{\perp}^{2}-r^{2}}}
\end{aligned}
$$

We obtain very interesting result: the normal force distribution in $3 \mathrm{D}\left[\frac{2}{3} s(r)+p(r)\right]$ is the Abel image of the light front shear force distribution $\frac{4}{\pi} \mathcal{S}\left(x_{\perp}\right)$ in 2D. Both Eq. (7) and Eq. (8) can be compactly written as:

$$
\begin{gathered}
\mathcal{S}\left(x_{\perp}\right)=x_{\perp}^{2} A[s]\left(x_{\perp}\right), \\
\frac{1}{2} \mathcal{S}\left(x_{\perp}\right)+\mathcal{P}\left(x_{\perp}\right)=\frac{1}{2} A\left[r^{2}\left(\frac{2}{3} s+p\right)\right]\left(x_{\perp}\right) \\
s(r)=-\frac{2}{\pi} r^{2} A\left[x_{\perp} \frac{d}{d x_{\perp}}\left(\frac{\mathcal{S}\left(x_{\perp}\right)}{x_{\perp}^{2}}\right)\right](r), \\
\frac{2}{3} s(r)+p(r)=\frac{4}{\pi} A[\mathcal{S}](r) .
\end{gathered}
$$

See Appendix for the explanation of the Abel transformation operation $A[\ldots](\ldots)$. The remarkable property of the Abel transformation is its positivity: the Abel image of a positive function is also a positive function. This is the key property for our analysis of the local stability criteria in the next subsections.

From Eq. (9) with help of integral relations for the Abel transformation (A5) it is easy to check that the 2D von Laue stability condition for the pressure:

$$
\int d^{2} \mathbf{x}_{\perp} \mathcal{P}\left(x_{\perp}\right)=0
$$

is satisfied automatically. This 2D stability condition was discussed for the first time in section 5.1.2 of [4]. Given the simple mathematical properties of the Abel transformation discussed in the Appendix, it is very easy to obtain number of other integral relations between 2D force distributions which are counterparts of the relations for 3D force distributions derived in Appendix A of Ref. [1]. Actually any integral relation for 3D force distributions obtained e.g. in Refs. $[1,4]$ can be immediately "translated" to the analogous relation for the light front force distribution. This demonstrates the power of the Abel transformation-one does not need to derive the equations for the 2D force distributions anew, they are already known in the literature. Below we discuss only the most important of them.

One of the important equation for $\mathcal{S}$ and $\mathcal{P}$ is the equilibrium equation:

$$
\mathcal{P}^{\prime}\left(x_{\perp}\right)+\frac{1}{2} \mathcal{S}^{\prime}\left(x_{\perp}\right)+\frac{1}{x_{\perp}} \mathcal{S}\left(x_{\perp}\right)=0,
$$

which can be immediately obtained from the $3 \mathrm{D}$ equilibrium equation with help of Eq. (A4) for the Abel transformation. 
The Druck-term can be expressed in terms of 2D force distributions as:

$D(0)=-m \int d^{2} x_{\perp} x_{\perp}^{2} \mathcal{S}\left(x_{\perp}\right)=4 m \int d^{2} x_{\perp} x_{\perp}^{2} \mathcal{P}\left(x_{\perp}\right)$.

Owing to the property of the Abel transformation (A5) the resulting Druck-term $D(0)$ coincides with that obtained from 3D Breit frame force distributions:

$$
D(0)=-\frac{4}{15} m \int d^{3} r r^{2} s(r)=m \int d^{3} r r^{2} p(r) .
$$

\section{A. Local stability conditions}

In Refs. [1,4,24] it was argued that for the stability of the mechanical system the 3D pressure and shear forces should satisfy the following inequality:

$$
\frac{2}{3} s(r)+p(r)>0 .
$$

From Eq. (9) we see immediately that this 3D stability condition implies:

$$
\frac{1}{2} \mathcal{S}\left(x_{\perp}\right)+\mathcal{P}\left(x_{\perp}\right)>0
$$

as the Abel image of a positive function is also positive. The above inequality is analogous to the $3 \mathrm{D}$ local stability conditions (15) because $\frac{1}{2} \mathcal{S}\left(x_{\perp}\right)+\mathcal{P}\left(x_{\perp}\right)$ corresponds to the distribution of normal force in $2 \mathrm{D}$. This local stability condition was discussed in Ref. [12]. We think it is important result that the stability conditions in 3D imply the stability of 2D mechanical system.

One can show that the 3D stability condition (15) can be obtained from the positivity of the shear force distribution (pressure anisotropy) $s(r)>0$. Indeed, from the 3D EMT conservation equation:

$$
\frac{d}{d r}\left(\frac{2}{3} s(r)+p(r)\right)=-\frac{2}{r} s(r)
$$

for $s(r)>0$ we conclude that $\frac{2}{3} s(r)+p(r)$ is a monotonically decreasing function. As this function at large distances goes to zero it must be positive. From Eq. (7) we see immediately that the condition $s(r)>0$ implies that:

$$
\mathcal{S}\left(x_{\perp}\right)>0, \quad \frac{1}{2} \mathcal{S}\left(x_{\perp}\right)+\mathcal{P}\left(x_{\perp}\right)>0 .
$$

So we see that the stronger 3D stability conditions $s(r)>0$ implies also analogous stronger condition in 2D. We note that to ensure the 3D stability condition (15) we need stronger stability condition in $2 \mathrm{D} \mathcal{S}\left(x_{\perp}\right)>0$, see Eq. (10).
The condition (16) is not enough to guarantee the stability in $3 \mathrm{D}$.

Using the positivity of the normal forces we can introduce the transverse mechanical radius:

$$
\begin{aligned}
&\left\langle x_{\perp}^{2}\right\rangle_{\text {mech }}=\frac{\int d^{2} x_{\perp} x_{\perp}^{2}\left(\frac{1}{2} \mathcal{S}\left(x_{\perp}\right)+\mathcal{P}\left(x_{\perp}\right)\right)}{\int d^{2} x_{\perp}\left(\frac{1}{2} \mathcal{S}\left(x_{\perp}\right)+\mathcal{P}\left(x_{\perp}\right)\right)} \\
& \stackrel{\text { Eq.(A5) })}{=} \frac{2}{3} \frac{\int d^{3} r r^{2}\left(\frac{2}{3} s(r)+p(r)\right)}{\int d^{3} r\left(\frac{2}{3} s(r)+p(r)\right)}=\frac{2}{3}\left\langle r^{2}\right\rangle_{\text {mech }} .
\end{aligned}
$$

Our result, which is two times smaller than the corresponding result of Ref. [12], corresponds to usual geometric ratio of $2 / 3$ between $2 \mathrm{D}$ and $3 \mathrm{D}$ mean square radii. The generalization of Eq. (19) for higher moments has the form:

$$
\left\langle x_{\perp}^{N}\right\rangle_{\text {mech }}=\frac{\Gamma\left(\frac{N+2}{2}\right) \Gamma\left(\frac{3}{2}\right)}{\Gamma\left(\frac{N+3}{2}\right)}\left\langle r^{N}\right\rangle_{\text {mech }} .
$$

\section{B. 2D pressure at the origin and the line tension}

Our physics intuition about the 2D light front force distributions is very limited. We shall see below in Sec. III on the example on very simple mechanical system-a liquid drop- that its light front force distributions have no vivid mechanical interpretation. The Abel $2 \mathrm{D} \leftrightarrow 3 \mathrm{D}$ relations derived here allow us to gain additional insights into the physics meaning and into visualization of the light front force distributions. For that we consider several basic quantities on the light front in terms of 3D quantities.

The 2D pressure in the centre of the nucleon can be easily obtained from Eq. $(7)^{3}$ :

$$
\mathcal{P}(0)=\int_{0}^{\infty} d r s(r)=\gamma^{(3 \mathrm{D})} .
$$

Here $\gamma^{(3 D)}$ has the meaning of the surface tension of the 3D mechanical system. This new relation provides us with elucidation of the physics meaning of the $2 \mathrm{D}$ pressure inside the hadron in terms of more intuitive 3D quantities. Obviously the 2D pressure in the centre satisfies Kelvin relation, generalized to $2 \mathrm{D}$ :

$$
\mathcal{P}(0)=\int_{0}^{\infty} \frac{d x_{\perp}}{x_{\perp}} \mathcal{S}\left(x_{\perp}\right)
$$

this relation was derived for the first time in Ref. [4]. Using the properties of the Abel transformation one can easily see that for the case of a singular normal force distribution in 3D the corresponding singularity of $2 \mathrm{D}$ counterpart is softer by one unit, for example $1 / r \rightarrow \ln \left(1 / x_{\perp}\right), 1 / r^{2} \rightarrow 1 / x_{\perp}$, etc.

\footnotetext{
${ }^{3}$ In our derivation we assume that $3 \mathrm{D} s(r)$ is not singular at $r=0$, which corresponds to nullification of $2 \mathrm{D} \mathcal{S}(0)$.
} 
The 2D line tension (2D counterpart of the surface tension in 3D) $\gamma^{(2 \mathrm{D})}$ can be obtained as:

$$
\gamma^{(2 \mathrm{D})}=\int_{0}^{\infty} d x_{\perp} \mathcal{S}\left(x_{\perp}\right)=\frac{\pi}{4} \int_{0}^{\infty} d r r s(r) .
$$

Again this is a new relation which provides us with the physics interpretation of the $2 \mathrm{D}$ line tension in terms of $3 \mathrm{D}$ shear force distribution.

\section{LIGHT FRONT FORCE DISTRIBUTIONS IN A LIQUID DROP}

We start with illustration of the $3 \mathrm{D} \leftrightarrow 2 \mathrm{D}$ relations from previous section on the example of a very simple mechanical system-a large nucleus as a liquid drop. Our aim here is twofold. First, with help of Abel transformation we obtain the 2D light front force distributions in a large nucleus. The knowledge of 2D light front force distributions can be very helpful for the developing of the models of nuclear generalized parton distributions (GPDs) and for phenomenology of hard exclusive process on nuclei. We note that, to our best knowledge, these 2D distributions can not be obtained by other methods. Our second motivation is to get more insight into physics interpretation of the light front force distributions. The simple and lucid mechanical system like a liquid drop perfectly serves this purpose.

For a large nucleus as a liquid drop 3D force distributions are vivid and intuitive: the shear forces are concentrated near the drop's surface and normal force is positive and constant in the drop's bulk:

$s(r)=\gamma^{(3 \mathrm{D})} \delta\left(R_{0}-r\right), \quad \frac{2}{3} s(r)+p(r)=\frac{2 \gamma^{(3 \mathrm{D})}}{R_{0}} \theta\left(R_{0}-r\right)$,

where $R_{0}$ is a drop's radius and $\gamma^{(3 \mathrm{D})}$ is the surface tension. Phenomenologically, $\gamma^{(3 \mathrm{D})} \simeq 1.0 \mathrm{MeV} / \mathrm{fm}^{2}$ for large nuclei obtained from Weizsäcker mass formula.

On the light front the corresponding force distributions are distorted due to motion of the observer and with help of Abel transformations (9) can be obtained in the following form:

$$
\begin{aligned}
\mathcal{S}\left(x_{\perp}\right) & =\frac{\gamma^{(3 \mathrm{D})}}{R_{0}} \frac{x_{\perp}^{2}}{\sqrt{R_{0}^{2}-x_{\perp}^{2}}} \theta\left(R_{0}-x_{\perp}\right), \\
\frac{1}{2} \mathcal{S}\left(x_{\perp}\right)+\mathcal{P}\left(x_{\perp}\right) & =\frac{\gamma^{(3 \mathrm{D})}}{R_{0}} \sqrt{R_{0}^{2}-x_{\perp}^{2}} \theta\left(R_{0}-x_{\perp}\right) .
\end{aligned}
$$

We see that, in contrast to 3D force distributions (24), the 2D light front force distributions do not have a vivid form. The 2D normal forces are not constant in the bulk of the drop and the 2D shear forces are not concentrated around the edge of the drop. We think that without use of the Abel transformation it would be very difficult to guess that $2 \mathrm{D}$ force distributions (25) correspond to a very simple mechanical system - the liquid drop.

For the developing of the models for nuclear GPDs it is important to know estimates for the basic numbers related to $2 \mathrm{D}$ forces, as well as their dependence on the atomic number $A$. The use of the Abel transformation allow us to obtain these estimates very easily. Using the results of previous section we obtain for the $2 \mathrm{D}$ pressure in the centre:

$$
\mathcal{P}(0)=\gamma^{(3 \mathrm{D})} \simeq 1.0 \frac{\mathrm{MeV}}{\mathrm{fm}^{2}},
$$

which is $A$ independent, i.e., it has qualitatively different dependence on the atomic number in comparison with $3 \mathrm{D}$ $p(0) \sim A^{-1 / 3}$. The $2 \mathrm{D}$ line tension (has dimension of the force) is

$$
\gamma^{(2 \mathrm{D})}=\frac{\pi}{4} R_{0} \gamma^{(3 \mathrm{D})} \simeq 0.88 A^{1 / 3} \frac{\mathrm{MeV}}{\mathrm{fm}} .
$$

It increases with the atomic number $A$, while the analogous quantity in $3 \mathrm{D}$ (surface tension) is independent of $A$. The calculated numbers and their $A$ dependences can be useful for a modeling of nuclear GPDs.

\section{LIGHT FRONT FORCE DISTRIBUTIONS IN THE NUCLEON AS CHIRAL SOLITON}

The large $N_{c}$ argumentation by Witten $[25,26]$ justified that $N_{c}$ quarks constituting a baryon can be considered in a mean (nonfluctuating) field that does not change as $N_{c} \rightarrow \infty$. In this picture the corresponding mean-field can be considered as a classical one, i.e., as a "chiral soliton."

The pioneering calculations of the pressure and shear force distributions in the large $N_{c}$ nucleon were performed in the chiral quark-soliton model [27] and in the Skyrme model in Refs. [28,29]. More recently these studies were extended to the nucleon in the nuclear matter [30-32], to $\Delta$-baryon $[24,33,34]$ and to the charmed baryons in Ref. [35]. As all these approaches employ the large $N_{c}$ picture the relativistic corrections are parametrically suppressed by $1 / N_{c}$, and all distributions can be obtained directly as functionals of the chiral mean-field.

We use here the results of Ref. [28] for 3D $s(r)$ and $p(r)$ to compute their light front counterparts using Eq. (7). The results are shown on Fig 1 where the light front shear force $\mathcal{S}\left(x_{\perp}\right)$ and pressure $\mathcal{P}\left(x_{\perp}\right)$ are shown in the left panel. On the right panel we show the results for the distribution of normal and transverse forces on light front. It is very interesting that the obtained light front distributions have the shapes very similar to their 3D counterparts (see, e.g., Figs. 3,4 of Ref. [28]). For example, the transverse force distribution has a node about the same position around $\sim 0.4 \div 0.5 \mathrm{fm}$ in both $3 \mathrm{D}$ and $2 \mathrm{D}$, also both $3 \mathrm{D}$ and $2 \mathrm{D}$ pressure distributions have nodes at about the same position 

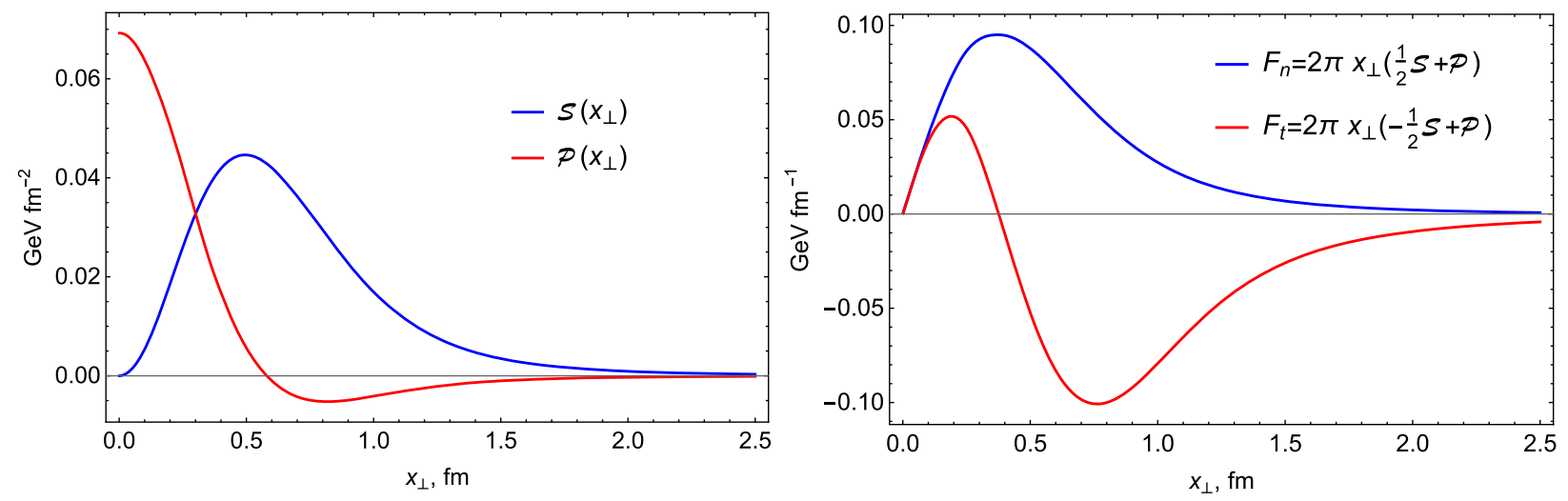

FIG. 1. The 2D pressure and share force distributions calculated in Skyrme model (left panel) and distribution of normal and tangential 2D forces calculated in the same model (right panel).

of $\sim 0.6 \div 0.7 \mathrm{fm}$. We see that in contrast to the liquid drop the reduction from $3 \mathrm{D}$ to $2 \mathrm{D}$ does not change the force distributions qualitatively. It would be interesting to understand the reason for that.

From the left panel of Fig. 1 one can easily read off the value of the $2 \mathrm{D}$ pressure $\mathcal{P}\left(x_{\perp}\right)$ in the nucleon center:

$$
\mathcal{P}(0) \simeq 70.0 \frac{\mathrm{MeV}}{\mathrm{fm}^{2}}
$$

It is instructive to compare it with the corresponding $2 \mathrm{D}$ pressure at the origin for a large nucleus given in (26). The line tension also can be easily computed with help of Eq. (23) with the result:

$$
\gamma^{(2 \mathrm{D})} \simeq 34.0 \frac{\mathrm{MeV}}{\mathrm{fm}}
$$

Again it is instructive to compare it with the result for a large nucleus (27). The 2D mean square mechanical radius is scaled by the geometric factor of $2 / 3$ relative to the $3 \mathrm{D}$ mechanical radius. One can show in the Skyrme model that $\left\langle x_{\perp}^{2}\right\rangle_{\text {charge }} /\left\langle r^{2}\right\rangle_{\text {charge }}=2 / 3$. Therefore, in $2 \mathrm{D}$ we obtain the same ratio of mechanical radius to charge one as in $3 \mathrm{D}$. The latter was estimated as $\simeq 0.75$ [1], hence we obtain:

$$
\frac{\left\langle x_{\perp}^{2}\right\rangle_{\text {mech }}}{\left\langle x_{\perp}^{2}\right\rangle_{\text {charge }}} \simeq 0.75 \quad[\text { Skyrme model }]
$$

Given the simplicity of the 3D $\rightarrow 2 \mathrm{D}$ reduction formula (7) the reader can easily obtain any other results for light front force distributions from the results of Ref. [28]. In general, the Abel transformation relations (9), (10) can be used to obtain 2D light front force distributions from any calculation of 3D Breit frame ones. For example, it is very easy to obtain large $x_{\perp}$ asymptotic of light front force distributions from the large $r$ asymptotic of $p(r)$ and $s(r)$ calculated in chiral EFT, see Eq. (26) of Ref. [18].

\section{CONCLUSIONS AND OUTLOOK}

We showed that the 2D light front force distributions and the 3D Breit frame ones are related to each other by invertible Abel transformation (9), (10). This finding establishes the mathematical equivalence of two types of force distributions. We proved an important property of the transformations (9), (10) - they do not change the positivity properties of the normal forces in $2 \mathrm{D}$ and 3D. It is a very important observation, it implies that the 3D local stability conditions for the force distributions automatically imply the stability of the corresponding 2D mechanical system. In this way we established not only mathematical equivalence of 2D and 3D forces distributions but also their physics equivalence in terms of stability. The beauty and simplicity of the $2 \mathrm{D} \leftrightarrow 3 \mathrm{D}$ transformations (9), (10) allowed us to derive a number of new relations for light front force distributions which were not known in the literature.

We discussed that the $2 \mathrm{D} \leftrightarrow 3 \mathrm{D}$ transformations can be used for calculations of the light front force distributions in various dynamical models for the hadron structure. Usually the direct calculation of 2D light front force distributions in the models is a very difficult (if not impossible) task. The Abel transformation can be used to compute 2D light front force distributions even in the models which are principally defined only in the rest frame of the hadron. This extends considerably the "toolkit" for studies of light front force distributions. To illustrate this, we computed 2D light front force distributions inside a large nucleus and in the nucleon using the liquid drop model and the Skyrme model correspondingly, thus providing the first dynamical calculation of the light front force distributions in the hadrons.

Another important field of possible applications of the derived Abel relations is the gaining a new insight into the mechanical interpretation of the 2D light front force distributions. The latter usually do not have vivid and intuitive mechanical interpretation. On contrary, the 3D force distributions usually have very simple and intuitive elucidation. Moreover, there are many theoretical methods to analyze mechanical properties of various physics system 
using the $3 \mathrm{D}$ force distributions in the rest frame of the considered system. The relations between 3D and 2D force distributions derived here allow us to apply these well developed methods to analysis of the light front force distributions.

It would be interesting to generalize formalism presented here to the case of nonspherically symmetric hadron (with spin $>1 / 2$ ). We expect that in more general case the Abel transformation is replaced by the radon one. This idea has been addressed but not fully investigated in [36].

\section{ACKNOWLEDGMENTS}

M. V. P. is grateful to Jambul Gegelia, Cédric Lorcé, and Peter Schweitzer for many illuminating discussions, and to Adam Freese for correspondence. This work was supported in part by the Deutsche Forschungsgemeinschaft (DFG, German Research Foundation) Project-ID 196253076 TRR 110, and by the BMBF (Grant No. 05P2018).

\section{APPENDIX: BASICS OF ABEL TRANSFORMATION}

For readers' convenience we give here the basic formulas for Abel transformation. For the function $g(r)$ its Abel transform (Abel image) $A[g](x) \equiv \mathcal{G}(x)$ can be written as:

$$
A[g](x) \equiv \mathcal{G}(x)=\int_{x}^{\infty} \frac{d r}{r} g(r) \frac{1}{\sqrt{r^{2}-x^{2}}} .
$$

The inverse transformation has the form [23]:

$$
g(r)=-\frac{2}{\pi} r^{2} \int_{r}^{\infty} d x \frac{d \mathcal{G}(x)}{d x} \frac{1}{\sqrt{x^{2}-r^{2}}} .
$$

From the above equations it is obvious the positivity property of the Abel transformation: Abel image of the positively defined function is also positively defined. Below we give number of relations which are useful for derivation of various properties of the light front distributions.

Inverse Abel transformation (A2) can be written as the direct Abel transformation:
TABLE I. Abel transforms of various functions.

\begin{tabular}{lc}
\hline \hline$g(r)$ & $A[g](x)$ \\
\hline$r^{2} e^{-\lambda r^{2}}$ & $\frac{1}{2} \sqrt{\frac{\pi}{\lambda}} e^{-\lambda x^{2}}$ \\
$r e^{-\lambda r}$ & $K_{0}(\lambda x)$ \\
$r \sin (\omega r)$ & $\frac{\pi}{2} J_{0}(\omega x)$ \\
$r \cos (\omega r)$ & $-\frac{\pi}{2} Y_{0}(\omega x)$ \\
$r K_{0}(\lambda r)$ & $\frac{1}{2} K_{0}\left(\frac{\lambda x}{2}\right)^{2}$ \\
$r K_{1}(\lambda r)$ & $\frac{\pi}{2} \frac{e^{-\lambda x}}{\lambda x}$ \\
$r J_{1}(\omega r)$ & $\frac{\sin (\omega x)}{\omega x}$ \\
$r Y_{1}(\omega r)$ & $-\frac{\cos (\omega x)}{\omega x}$ \\
$\theta\left(R_{0}-r\right)$ & $\frac{1}{x} \arccos \left(\frac{x}{R_{0}}\right) \theta\left(R_{0}-x\right)$ \\
$r^{2} \theta\left(R_{0}-r\right)$ & $\sqrt{R_{0}^{2}-x^{2} \theta\left(R_{0}-x\right)}$ \\
\hline \hline & \\
$A^{-1}[\mathcal{G}(x)](r)=-\frac{2}{\pi} r^{2} A\left[x \frac{d}{d x} \mathcal{G}(x)\right](r)$. & $(\mathrm{A} 3)$
\end{tabular}

Derivative of the Abel image:

$$
\frac{d}{d x} A[g](x)=x A\left[r \frac{d}{d r} \frac{g(r)}{r^{2}}\right]
$$

Integrals of the Abel image:

$$
\int d^{2} x_{\perp} x_{\perp}^{\alpha} A[g]\left(x_{\perp}\right)=\frac{\sqrt{\pi}}{4} \frac{\Gamma\left(\frac{\alpha+2}{2}\right)}{\Gamma\left(\frac{\alpha+3}{2}\right)} \int d^{3} r r^{\alpha-2} g(r) .
$$

We give also the Abel images of some basic functions, they can be useful for practical applications, for example:

$$
A\left[\frac{1}{r^{\alpha}}\right](x)=\frac{\sqrt{\pi}}{2} \frac{\Gamma\left(\frac{\alpha+1}{2}\right)}{\Gamma\left(\frac{\alpha+2}{2}\right)} \frac{1}{x^{\alpha+1}},
$$

can be used to obtain large $x_{\perp}$ asymptotic of light front force distributions from the large $r$ asymptotic of $p(r)$ and $s(r)$ obtained in chiral EFT, see Eq. (26) of Ref. [18]. Further useful Abel images are collected in Table I.
[1] M. V. Polyakov and P. Schweitzer, Int. J. Mod. Phys. A 33, 1830025 (2018).

[2] M. V. Polyakov, Phys. Lett. B 555, 57 (2003).

[3] C. Lorcé, Phys. Rev. Lett. 125, 232002 (2020).

[4] C. Lorce, H. Moutarde, and A. P. Trawinski, Eur. Phys. J. C 79, 89 (2019).

[5] C. Lorcé, L. Mantovani, and B. Pasquini, Phys. Lett. B 776, 38 (2018).
[6] E. P. Wigner, Phys. Rev. 40, 749 (1932).

[7] M. Hillery, R. F. O'Connell, M. O. Scully, and E. P. Wigner, Phys. Rep. 106, 121 (1984).

[8] D. R. Yennie, M. M. Levy, and D. G. Ravenhall, Rev. Mod. Phys. 29, 144 (1957).

[9] M. Burkardt, Phys. Rev. D 62, 071503 (2000); 66, 119903 (E) (2002).

[10] G. A. Miller, Annu. Rev. Nucl. Part. Sci. 60, 1 (2010). 
[11] R. L. Jaffe, Phys. Rev. D 103, 016017 (2021).

[12] A. Freese and G. A. Miller, arXiv:2102.01683v1.

[13] Y. B. Zeldovich and I. D. Novikov, Relativistic Astrophysics (University of Chicago Press, Chicago, 1971), Vol. 1.

[14] L. Herrera and N. O. Santos, Phys. Rep. 286, 53 (1997).

[15] I. Y. Kobzarev and L. B. Okun, Zh. Eksp. Teor. Fiz. 43, 1904 (1962) [Sov. Phys. JETP 16, 1343 (1963)].

[16] H. Pagels, Phys. Rev. 144, 1250 (1966).

[17] S. Cotogno, C. Lorce, P. Lowdon, and M. Morales, Phys. Rev. D 101, 056016 (2020).

[18] H. Alharazin, D. Djukanovic, J. Gegelia, and M. V. Polyakov, Phys. Rev. D 102, 076023 (2020).

[19] V. D. Burkert, L. Elouadrhiri, and F. X. Girod, Nature (London) 557, 396 (2018).

[20] K. Kumericki, Nature (London) 570, E1 (2019).

[21] H. Dutrieux, C. Lorcé, H. Moutarde, P. Sznajder, A. Trawiński, and J. Wagner, Eur. Phys. J. C 81, 300 (2021).

[22] S. Kumano, Q. T. Song, and O. V. Teryaev, Phys. Rev. D 97, 014020 (2018).

[23] N. H. Abel, J. für die Reine und Angew. Math. 1, 153 (1826); For application of Abel tomography to hard exclusive processes see: A. M. Moiseeva and M. V. Polyakov, Nucl. Phys. B832, 241 (2010); M. V. Polyakov, Phys. Lett. B 659, 542 (2008).
[24] I. Perevalova, M. Polyakov, and P. Schweitzer, Phys. Rev. D 94, 054024 (2016).

[25] E. Witten, Nucl. Phys. B160, 57 (1979).

[26] E. Witten, Nucl. Phys. B223, 433 (1983).

[27] K. Goeke, J. Grabis, J. Ossmann, M. V. Polyakov, P. Schweitzer, A. Silva, and D. Urbano, Phys. Rev. D 75, 094021 (2007).

[28] C. Cebulla, K. Goeke, J. Ossmann, and P. Schweitzer, Nucl. Phys. A794, 87 (2007).

[29] K. Goeke, J. Grabis, J. Ossmann, P. Schweitzer, A. Silva, and D. Urbano, Phys. Rev. C 75, 055207 (2007).

[30] H. C. Kim, P. Schweitzer, and U. Yakhshiev, Phys. Lett. B 718, 625 (2012).

[31] J. H. Jung, U. Yakhshiev, and H. C. Kim, J. Phys. G 41, 055107 (2014).

[32] J. H. Jung, U. Yakhshiev, H. C. Kim, and P. Schweitzer, Phys. Rev. D 89, 114021 (2014).

[33] J. Y. Panteleeva and M. V. Polyakov, Phys. Lett. B 809, 135707 (2020).

[34] J. Y. Kim and B. D. Sun, Eur. Phys. J. C 81, 85 (2021).

[35] J. Y. Kim, H. C. Kim, M. V. Polyakov, and H. D. Son, Phys. Rev. D 103, 014015 (2021).

[36] W. Cosyn, S. Cotogno, A. Freese, and C. Lorcé, Eur. Phys. J. C 79, 476 (2019). 